\title{
Herramientas digitales que apoyan en la toma de decisiones de las mipymes en México
}

\section{Digital tools that support decision making for MSMEs in Mexico}

\author{
DOI: $10.46932 / \operatorname{sjdv} 3 n 1-032$
}

Received in: Dec 30st, 2021

Accepted in: Jan 1th, 2022

\section{M.E. Alma Elizabeth Miranda Quiñones}

Profesora de Gestión empresarial en el Instituto Tecnológico de Tlalnepantla del Tecnológico Nacional de México, Tlanepantla, Estado de México.

E-mail: almaelizabeth2@gmail.com

\section{M.A.N. Ociel Núñez Sánchez}

Profesor de Plan de Negocios en el Instituto Tecnológico de Tlalnepantla del Tecnológico Nacional de México, Tlalnepantla, Estado de México

E-mail: ocielns@tlalnepantla.tecnm.mx

\section{M.E. María Magdalena Trejo Lorenzana}

Profesora de Planeación Financiera en el Instituto Tecnológico de Tlalnepantla, del Tecnológico Nacional de México, Tlalnepantla, Estado de México

E-mail: maría.tl@tlalnepantla.tecnm.mx

\section{RESUMEN}

Ante la problemática de la perdida de gran cantidad de micro, pequeñas y medianas empresas (Mipymes), por la emergencia sanitaria COVID-19 y la falta de conocimiento y recursos digitales, se presenta un documento que describe ocho herramientas tecnológicas, mostrando las ventajas que cada una de ellas proporciona a este grupo de empresas, se lleva a cabo un estudio exploratorio, a través de una revisión documental sobre el tema, la información obtenida acerca de las herramientas se encuentran estructuradas, en tres aspectos; para apoyar en el resguardo e intercambio de información; como aportación en la mejora de los procesos y en función de la atención a clientes; lo que permite a los responsables de la toma de decisiones, identificar con claridad las ventajas que le pueden ofrecer el conjunto de herramientas tecnológicas presentadas en el presente documento.

Palabras clave: herramientas tecnológicas, digitalización, Mipymes, gestión empresarial, toma de decisiones.

\section{ABSTRACT}

Given the problem of the loss of a large number of micro, small and medium enterprises (MSMEs), by the health emergency COVID-19 and the lack of knowledge and digital resources, a document is presented that describes eight technological tools, showing the advantages that each of them provides to this group of companies, an exploratory study is carried out through a documentary review on the subject, the information obtained about the tools are structured in three aspects; The information obtained about the tools are structured in three aspects: to support in the storage and exchange of information; as a contribution to the improvement of processes and in terms of customer service; which allows decision makers to clearly identify the advantages that the set of technological tools presented in this document can offer. 
Keywords: technological tools, digitalization, MSMEs, business management, decision making.

\section{INTRODUCCIÓN}

Los recursos electrónicos vinieron a revolucionar los ambientes económico, social y político; y sin duda en los tiempos actuales en que se vive la pandemia por COVID-19, se convirtieron en la alternativa de mayor impacto en la estancia y subsistencia de muchas de las organizaciones, sin embargo, existe la problemática de acuerdo con ferruzola (2011) citado en Hallanca 2019, que hace falta conocimiento y recursos en el tema de las tecnologías de la información y comunicaciones (TIC's), por lo que se dificulta visualizar el impacto y beneficios que pueden lograrse a corto plazo, tomando en cuenta que de abril a agosto de 2020, se han perdido 320,000 micro, pequeñas y medianas empresas (Mipymes) en México, de acuerdo con los datos de la Asociación Latinoamericana de Micros, Pequeños y Medianos Empresarios (Alampyme), y en contraparte de acuerdo a los datos del censo emitido en 2020 por el Instituto Nacional de Estadística, Geografía e Informática (INEGI, 2020) surgieron durante el periodo de la pandemia, 619,443 negocios, por lo que algunas asociaciones están tratando de fortalecer a este grupo de organizaciones guiándolas hacia la digitalización (Martínez, 2020).

La prioridad se centra en lograr que las Mipymes, salgan avante en esta crisis pandémica, por lo que los gobiernos generaron programas orientados a la capacitación y sensibilización en el uso de las tecnologías de la información, para "promover el mercado local, brindar información de mercado y vincular los pequeños negocios con consumidores y socios estratégicos” (Heredia y Dini, 2021, p.16) priorizando la reducción de costos y mejorar la productividad para hacerlas más flexibles, como fue el caso del sector educativo donde de acuerdo con Argüello y Espinoza (2021), exponen, como las herramientas digitales asociadas con los negocios demostraron en este periodo de contingencia, ser un aliado no tanto para generar competitividad sino para continuar activos en el mercado.

El tiempo fue demasiado corto para elegir el recurso más adecuado, aun en estos momentos de la nueva normalidad, algunos negocios siguen luchando por encontrar el recurso tecnológico que sea de fácil acceso, además de contar con la flexibilidad de adaptarse a las necesidades, condiciones y presupuestos disponibles, correspondiendo al tomador de decisiones, la función de realizar la selección de las estrategias digitales, que se adecue a las características de la organización (Apolo-Romero et al., 2021) desde este punto de partida se considera importante 1. Identificar las herramientas tecnológicas que pueden apoyan en la toma de decisiones de las Mipymes en México, 2. Determinar la información que proporciona a la organización, 3. Mostrar las ventajas que otorga su implementación en la Mipyme. 
Desde este enfoque se ha integrado el documento con la intensión de describir de forma sencilla y concisa ocho herramientas factibles de ser incorporadas a las Mipymes, que pueden facilitar, suprimir tiempos y costos en sus procesos, mejorar la atención a sus clientes, vincularse con otras empresas o reducir el impacto ambiental como lo menciona Chávez y Roche, (2022), incluso incrementar su competitividad a través de la implementación del uso de las tic's como un mecanismo estratégico para la medición de su desempeño (Zapata, Arango y Adarme, 2010), fortalecer a este grupo empresarial para reducir la perdida de estos motores productivos y de servicios, que representan una gran relevancia dentro de la economía mexicana, confrontando los retos que ha traído la emergencia sanitaria, redireccionando gran parte de las actividades administrativas empresariales al ambiente digital, para conectar con un mayor número de clientes, pero al mismo tiempo reducir tiempos y costos, sin descuidar las actividades inherentes a la organización.

\section{METODOLOGÍA}

Se presenta una investigación aplicada dado que integra una propuesta de solución a un problema para la toma de decisiones dentro de las Mipymes (Vara Horna, 2012), con enfoque cualitativo, porque tiene la intención de conocer la realidad social respecto al problema presentado (Álvarez-Gayou, 2003) y se basa en datos e información de investigaciones previas (Hernández-Sampieri, et al., 2016), por lo que es de tipo documental ya que de acuerdo a Cazarez, et al (1995) en Bernal (2014), se fundamenta en datos obtenidos de diversos documentos, de corte exploratorio al presentar una visión aproximada sobre el tema, que facilite la obtención de datos para precisar con claridad la información (Arias, 2012).

La construcción del documento se encuentra direccionada por tres aspectos, la descripción de la herramienta, su aplicabilidad y las ventajas que proporciona, por lo que se propone una estructura en tres dimensiones: a). Como captura y transferencia de información; b). Para reporte de información y c). Como sistema de aplicación de conocimiento, en donde se encuentran incorporadas diversas herramientas digitales, realizando la descripción de cada una, su aplicación dentro de las organizaciones, y ventajas que puede tener el seleccionarla, con la perspectiva de crear una guía que le facilite a la persona responsable el tomar la decisión de elegir la herramienta que se adecue a los requerimientos y características de la organización, y que al mismo tiempo le permita flexibilizarse y darle mayor dinamismo a su operación.

\section{DESARROLLO}

\subsection{LAS TECNOLOGÍAS DE LA INFORMACIÓN EN LA TOMA DE DECISIONES}

De acuerdo a la Organización de las Naciones Unidas (ONU), las tecnologías de la información son recursos informáticos que proporcionan un conjunto de actividades y soluciones (Zapata, et al., 2010), para apoyar en los procesos administrativos, en la organización de operaciones o en la logística, utilizando 
datos históricos, para administrar el presente y planear el futuro (Paletta y Dias, 2008), con la intención de reducir la incertidumbre en el proceso de fabricación de productos y/u otorgamiento de servicios (Velasteguí y Toaquiza, 2017), los sistemas digitales tienen el propósito de atender la demanda de información, al interior de las organizaciones para ayudar a contar con el conocimiento necesarios para llevar a cabo una buena toma de decisiones.

Asimismo, el uso de las herramientas digitales apoyan en la economía y reducción de consumibles, al integrarse un sistema de comunicación vía intranet permite agilizar el intercambio de mensajería al interior de la organización con mayor fluidez y rapidez, se minimiza el uso de papelería ya no tan necesaria y la información queda a resguardo en bases de datos electrónicas, asegurando el requerimiento de menor espacio, protección de documentos y una aportación hacia la sustentabilidad, con miras de incorporarse a las empresas de responsabilidad social (Chávez y Roche, 2022)

Diversos autores han planteado catalogar las herramientas tecnológicas y su función dentro de la organización, pero tomando en cuenta lo comentado por Kruise et al., (2016) respecto a que existen en el mercado una gran cantidad de softwares que no logran adaptarse en su totalidad a las necesidades de las empresas, se considera identificar las herramientas tecnológicas con mayor posibilidad de ajustarse a los requerimientos de las Mipymes en las condiciones actuales, así el presente documento, se ha integrado tomando en consideración las áreas estratégicas, como se muestra en la tabla 1, su desarrollo se ha establecido a partir de tres aspectos importantes, a). La captura y transferencia de información, b). Reporte de información, c). Como sistema de aplicación de conocimiento; aunque efectivamente existen una gran cantidad de sistemas y aplicaciones digitales que apoyan a los procesos administrativos, se han considerado los propuestos a partir de su aportación, costo y accesibilidad.

Tabla 1. Tipos de herramientas tecnológicas, según su función

\begin{tabular}{|c|c|c|}
\hline Categoría & Herramienta & Función en la organización \\
\hline \multirow[b]{4}{*}{$\begin{array}{l}\text { Captura y transferencia de } \\
\text { información }\end{array}$} & Internet & $\begin{array}{l}\text { Comunicación de datos globales a través de las (internet } \\
\text { protocol) IP }\end{array}$ \\
\hline & Banda ancha & Mecanismo de comunicación interna rápido y de bajo costo \\
\hline & $\begin{array}{l}\text { Intercambio electrónico de datos } \\
\text { (EDI) }\end{array}$ & $\begin{array}{l}\text { Transmisión de datos electrónicos entre empresas evitando } \\
\text { el uso de papel. }\end{array}$ \\
\hline & XML (eXtensible Markup Language) & $\begin{array}{l}\text { Apoya a los grupos de trabajo, haciendo enlaces } \\
\text { individuales dentro de la Web, pudiendo ser compartidos } \\
\text { con todos los integrantes. }\end{array}$ \\
\hline \multirow[b]{2}{*}{ Reporte de información } & Enterprise resource planning (ERP) & $\begin{array}{l}\text { Sistema de información gerencial que atiende operaciones } \\
\text { de producción y distribución de la empresa }\end{array}$ \\
\hline & $\begin{array}{l}\text { Sistema de administración de las } \\
\text { relaciones con el cliente (CRM) }\end{array}$ & Gestiona y administra el proceso de atención a clientes \\
\hline
\end{tabular}




\subsection{INTERNET}

Conocido también como la autopista de la comunicación, es una herramienta que proporciona un acercamiento a información basta en cualquier tópico que se desee atender, facilitando el acceso a datos globales y pertinentes que ayudan a la toma de decisiones (Campos-Sánchez, et al. 2021), como por ejemplo el conocer acerca de las estrategias que están tomando algunas pymes para llegar a sus clientes a través de las redes sociales, el servicio de entrega a domicilio o el préstamo de servicios de atención a través de videollamadas, esta herramienta es contratada por medio de paquetes de telefonía.

\subsection{BANDA ANCHA}

Es la velocidad con la que se puede navegar en internet, así como el tiempo que tarda en descargar información, los métodos o procesos que se encuentran disponibles en la actualidad, para mejorar la velocidad son: la conexión por cable, la fibra óptica, los datos móviles, el servicio satelital, entre otros.

El XML (eXtensible Markup Language)

El XML, lenguaje de resaltado extendido, es una herramienta de recuperación de información electrónica, cuando el volumen es excesivo, o se desea conocer la relevancia de los datos presentados, permiten elevar el conocimiento para apoyar a que la toma de decisiones sea más certera, al contar con información oportuna y precisa (Cabarcas, et al., 2014), la entrega de facturas en la actualidad se encuentran en formato PDF y XML.

\section{El EDI (Electronic Data Interchange)}

El intercambio electrónico de datos EDI, facilita una intercomunicación entre empresas de datos o información como es el caso de facturas u órdenes de compra, para facilitar el intercambio y evitar errores humanos, se ha establecido una estandarización en el EDI, fijados por la (American National Standard Institution) ANSI, aunque es un proceso de bajo costo, el internet ha sido adoptado por mayor número de empresas (Zapata, et al, 2010).

El servicio de internet y la Banda Ancha, van de la mano, el primero facilita el acceso a datos y el segundo es la velocidad con que se tiene acceso a la información, uniéndoseles las redes sociales, que hoy día se ha convertido en las herramientas favoritas de un gran número de pequeños empresarios, que utilizaron estos mecanismos para ofertar sus productos, dando continuidad a sus actividades comerciales a través del mercado electrónico.

\section{El sistema ERP (Enterprise resource planning)}

La planificación de los recursos empresariales (ERP), es un sistema de información asociado con la producción y distribución de productos, que conduce hacia un sistema estandarizado ya que se enlaza a una base de datos, centralizando la información de compras, ventas, distribución y logística, el beneficio 
que otorga a la empresa es el contar con información cruzada en todos los sentidos en el momento oportuno.

Los sistemas ERP están diseñados para una gestión integral, involucrando a todas las áreas, compartiendo información que facilite la eliminación de los datos innecesarios y al mismo tiempo, se tenga la posibilidad de realizar adecuaciones durante el proceso (Quiles, s.f.)

De las aplicaciones que tiene un ERP es que la información que contiene el sistema permite resolver problemas contables o fiscales, a través del control de inventarios, liquidez o pago de impuestos, los procesos de fabricación, la gestión del almacén, la gestión del abastecimiento.

Como parte del ERP se encuentra el intercambio electrónico de datos (EDI), que hace posible la comunicación de documentos entre clientes y proveedores y la facturación y banca electrónica; para el manejo de personal se encuentra el lean recruitment que básicamente agiliza el proceso de selección de personal, firma de contratos y comunicación on line (Quiles, s.f.).

Los beneficios que otorga a la organización esta herramienta son:

Optimizar, estandarizar y agilizar los procesos del negocio

Acceso a la información de manera inmediata y a todos los interesados

Compartir la información entre varias áreas de la organización

Disminuye el re-trabajo

Reduce costos

Elimina la improvisación

Este tipo de herramientas puede ayudar a eficientar los procesos en las pequeñas y medianas empresas, sin embargo, para incorporarlo a la organización se requiere de una mediana inversión, por lo que se sugiere, considerarlo en la planeación estratégica para ser considerado en el presupuesto a un corto o mediano plazo.

\section{El CRM (Customer Relationship Management)}

El Sistema para la administración de la relación con los usuarios (CRM), se centra en "potencializar la relación con el cliente, identificando segmentos de clientes que comparten perfiles similares, adecuando su oferta de servicios y/o productos a estos grupos" (Segarra, 2019, p.4), concentrándose en conocer sus necesidades y hábitos de compra, personalizar la interacción y crear campañas de marketing específicas, para incursionar en el mercado digital.

Los beneficios que aporta a la organización la herramienta del CRM son:

Alineamiento de las funciones departamentales con las necesidades del cliente

Identifica áreas de oportunidad para incrementar las ventas

Incrementa la satisfacción en el cliente 


\section{El WMS (Warehouse management system)}

El sistema de gestión de almacenamiento (WMS), es una herramienta tecnológica que apoya en la reducción de costos y control del manejo del almacenaje o el armado de pedidos (picking), dividiendo la zona de almacenamiento en procesos a través de contenedores, por ejemplo, las entradas y las salidas, al utilizar la automatización se modelan procesos y flujos de trabajo, para optimizar los tiempos en esta parte de la organización.

Sus ventajas se encuentran en la eliminación de posibles errores humanos, la optimización de los tiempos, y la mejora en los flujos de trabajo, manteniendo un control sobre las entradas, permanencia y salidas de los productos.

\section{El six sigma}

Six sigma es otro de los sistemas más completos para ser adoptados por la organización, ya que brinda soporte en el desarrollo de productos, adquisición de nuevos negocios, servicio al cliente, contabilidad y otras funciones empresariales.

A diferencia de un ERP es que six sigma establece sus parámetros de evaluación para identificar los resultados, a través de una métrica establecida en sigma que es una expresión estadística que representa a la desviación estándar, así seis sigma representa que indica \pm 3 desviaciones de la media que representan el $99.9 \%$ de probabilidad de que ocurra un evento, conduciendo a establecer límites de calidad en la manufactura, o en la prestación de un servicio.

Un ejemplo es el presentado por Globus Biogestion (2016), en que presenta los puntos críticos de su producto, cuadernos escolares, centrados en el blanco de la hoja, el encuadernado y la definición de la cuadricula:

Teniendo que con 3 sigma representa $(0.933) *(0.933) *(0.933)=0.8121$

Lo que significa que por cada 100 cuadernos saldrán 18.79 defectuosos en cualquiera de los tres aspectos (El blanco de la hoja, la encuadernación o la definición de la cuadricula).

Con 6 sigma se tendría $(0.9999) *(0.9999) *(0.9999)=0.9997$

Lo que significa que por cada 100 cuadernos saldrán 0.03 defectuosos

La intención del sistema es la eliminación de errores u obstáculos en el proceso de producción o servicio otorgado, sustentado en su calidad, este sistema es factible de aplicarse en todos los procesos no solo en la producción, sino que puede ser adaptado en cualquiera de las partes de su proceso.

\section{COMENTARIOS FINALES}

El covid-19 se presenta como un acelerador en la aceptación de la TIC's en todos los ambientes sobre todo en las Mipymes, el aislamiento social permitió que gran parte de las funciones empresariales encontraran su camino a través de las herramientas tecnológicas (Campos-Sánchez et al., 2021), la 
digitalización de las Mipymes en la actualidad resulta de gran relevancia, ya que pueden apoyar a flexibilizar los procesos y atender la problemática de subsistencia en el mercado que se encuentran viviendo, aunque en otro sentido se facilita la incorporación al ámbito de la responsabilidad social al reducir el consumo de recursos naturales, y emisión de documentos que hoy por hoy pueden ser emitidos y resguardados en bases de datos electrónicas, (Chávez y Roche, 2022), pero también se presenta el fenómeno de creación de nuevas empresas como es el caso de las fiestas a través de la aplicación de zoom (Sulbarán, 2020), atención psicológica, incluso las de asesoría sobre la digitalización (El país, 2021) atendiendo así la dificultad para la supervivencia y al mismo tiempo incorporando a la organización en un nuevo y actual amiente de desarrollo.

Son tres actividades a las que debe ponerse atención, principalmente para una adecuada toma de decisiones en una organización según Cabarcas, et al. (2014); en la entrada de insumos, el proceso y la salida de producto o servicio, de ahí que sea importante conocer las características y ventajas de cada una de las herramientas tecnológicas que pueden ayudar de forma integral a las organizaciones sobre todo cuando los recursos son escasos, con la misión de generar valor organizacional, que en palabras de Ward y Peppard, (2002) en Rodríguez y Lamarca (2012), debe permitir la toma de decisiones correcta; eficientar los procesos y sus resultados; contar con una comunicación inmediata y suscinta de los resultados obtenidos; mantener los datos históricos de la empresa; e incrementar la productividad de operativos y sus gestores, p. 16 para atender con rapidez los cambios, como los que se requirieron por la emergencia que se vivió a inicios de 2020 , . efectivo

Desde esta perspectiva se presenta una propuesta en la cual se estructuraron las herramientas digitales en función de cinco aspectos, 1. En el acopio y manejo de la información, 2. En cuanto al reporte que otorga la información que se presenta, 3. En relación con el conocimiento que se requiere para su realización, 4. En función de la accesibilidad y costo de aplicación, pero sobre todo 5. Considerando el aporte que proporcionan cada una de ellas al interior de la organización, con lo que se pretende mostrar la accesibilidad y facilidad de implementación, la sencilles para su operabilidad, además de contar con un sistema seguro de acopio de información, que puede ser susceptible de contar con intercambio entre organizaciones, traslado de documentos generado por los procesos, para atención a las necesidades de los clientes o simplemente para control de resultados de la productividad.

La elección de la herramienta tecnológica, que es factible de incorporar a la organización debe estar en función a las necesidades internas y externas de la empresa, sus características, el producto y/o los servicios prestados, por lo que el conocimiento de la utilidad y beneficios que aporta cada una de ellas, es de suma importancia al momento de tomar la decisión de su incorporación. 


\section{CONCLUSIONES}

Se identificaron ocho herramientas digitales que pueden apoyar a las Mipymes en su toma de decisiones, las cuales son susceptibles de ser incorporadas a la organización con alta facilidad, a costos accesibles y que representan una dificultad mínima para su operación, pese a que existen una gran cantidad y diversidad de herramientas se concluyó que las integradas en la presente propuesta, representan una mayor aportación al sector empresarial al que va dirigido.

En cuanto a la información que proporcionan las herramientas seleccionadas a la organización, se encuentran centradas en tres momentos, el proceso de acogida de la información, en su gestión y en la forma en que pueden integrarse a las funciones propias de la empresa, se muestra el funcionamiento de cada una de ellas, lo que facilita la detección e interpretación en el sentido de la utilidad que representan para la organización y la posibilidad para su incorporación en las Mipymes.

Las ventajas que proporciona la implementación en las Mipymes se ven reflejadas en gran medida en la capacidad que le otorga del conocimiento de manera veraz y oportuna sobre los cambios, económicos, técnicos, políticos e incluso culturales que se desarrollan en el entorno, lo que le permite al responsable de la toma de decisiones redireccionar la misión y visión empresarial.

Un aspecto que está cobrando fuerza y que permite a las Mipymes irse fortaleciendo es lograr hacer alianzas con otras empresas, en este sentido las herramientas digitales son un apoyo fundamental para facilitar el intercambio de información entre empresas reduciendo el tiempo de comunicación, acortando las distancias, evitando la necesidad de traslados continuos.

Otra ventaja que se puede observar es el ampliar la capacidad para atraer y retener clientes, ya que se tiene la posibilidad de incorporar sistemas que permiten el monitoreo dentro de su proceso mercantil lo que beneficia no solo a la organización, sino que mejora la atención al cliente, ayudando a generar una fidelización y proyección hacia nuevos mercados.

Cuando se es una empresa pequeña, lo que se busca es contar con información clara concisa y precisa, porque depende de ello para seguir avanzando y creciendo, especialmente las herramientas tecnológicas tienen la facilidad del manejo de datos, su protección, pero también su diseminación, lo que le permite concentrarse en la información de mayor impacto para el desarrollo y desempeño de la organización. 


\section{REFERENCIAS}

Álvarez-Gayou, J.L. (2003). Como hacer investigación cualitativa, fundamentos y metodología. Editorial Paidos

Apolo-Romero, J., Garzón, V., Quezada, J. y Prado, E. (2021). Aplicabilidad de inteligencia de negocio como soporte en toma de decisiones en la asociación bananera fincas de el oro. South Florida Journal of Development, 2(4), 5593- 5605. DOI: 10.46932/sfjdv2n4-048

Argüello, D. y Espinoza, C. (2021). El impacto de la era digital y su importancia en los negocios educativos en tiempos de Covid-19, South Florida Journal of Development, 2(5), 7446-7450. DOI: $10.46932 /$ sfjdv2n5-081

Arias, F. (2012). El proyecto de investigación, introducción a la metodología científica. (6 ${ }^{\mathrm{a}}$. Ed.) Editorial Episteme.

Bernal, C. (2016). Metodología de la investigación Administración, economía, humanidades y ciencias sociales $\left(4^{\mathrm{a}}\right.$. edición). Editorial Pearson Educación.

Cabarcas, A., Puello, P. y Martelo, J. (2014). Sistemas de información soportado en recuperación XML para pequeñas y medianas empresas (Pyme) de Cartagena de Indias, Colombia. Información Tecnológica, 26(2), 135-144. Disponible en: https://scielo.conicyt.cl/pdf/infotec/v26n2/art16.pdf

Campos-Sánchez, S., Cedeño-Palacios, C., Palma-García, Y. y Campos-Sánchez, V. (2021). Los negocios digitales y su importancia en la era actual. Revista Científica Dominio de las Ciencias, 7(1), [Especial], 1029-1043. DOI: http://dx.doi.org/10.23857/dc.v7i1.1755

Chávez, K. y Roche, M.L. (2022). La ética en la responsabilidad social empresarial. South Florida Journal of Development, 3(1), 177-193. DOI: 10.46932/sfjdv3n1-014

El país. (20 febrero 2021). Las empresas de salud y tecnología nacidas en la pandemia. El país Disponible en: https://cincodias.elpais.com/cincodias/2021/02/19/fortunas/1613763672_086612.html

Globus biogestion (2016). Seis Sigma fundamentos. [Video]. Youtube. Disponible en https://www.youtube.com/watch?v=eG-OZQspYUk

Heredia Zurita, A. y Dini, M. (2021). Análisis de las políticas de apoyo a las pymes para enfrentar la pandemia de COVID. 19 en Amériva Latina, [Documentos de Proyectos (LC/TS.2021/29)], Santiago, Comisión Económica para América Latina y el Caribe (CEPAL). Disponible en: https://repositorio.cepal.org/bitstream/handle/11362/46743/1/S2100104_es.pdf

Hernández-Sampieri, R., Fernández-Collado, C. y Baptista-Lucio, P. (2014). Metodología de la investigación ( $6^{\mathrm{a}}$ edición), Editorial McGraw Hill.

Huallanca, J. (2019). Análisis de uso de tecnología de información y su influencia en el crecimiento económico en la mediana y pequeña empresa en la ciudad de Chibcha, 2019. [Tesis de Maestría].

Instituto Nacional de Estadística, Geografía e Informática (INEGI). (2 diciembre de 2020). El INEGI presenta resultado de la segunda edición del ECOVID-IE y del resultado sobre la demografía de los negocios 2020.

(Comunicado

de

prensa

617/20). 
https://inegi.org.mx/contenidos/saladeprensa/boletines/2020/OtrTemEcon/ECOVIDIE_DEMOGNEG.pdf

Universidad

Autónoma

de

ICA.

Disponible

http://repositorio.autonomadeica.edu.pe/handle/autonomadeica/559

Kruize, J.W., Wolfert, J. Scholten, H., Verdouw, C.N., Kassahum, A. y Beulens. A. (2016). Una Arquitectura de referencia para ecosistemas de software agrícola. Computers and electronics in agricultura. 125, 12-28. DOI: https://doi.org/10.1016/j.compag.2016.04.011

Paletta, F., y Dias, N. (2008). Information technology and communication and best practices in it lifecycle management. Journal of technology management \& innovation, 3(4), 80-94. DOI. https://dx.doi.org/10.4067/S0718-27242008000200007

Quiles, J. (s.f.). Digitalización de los procesos administrativos de la pyme. Expertos en transformación digital. Clave i.

Rodríguez, J. y Lamarca, I. (2012). Gestión de la información y el conocimiento. Universidad Oberta de Cataluña.

Segarra, A. (2019). Desarrollo de un modelo de fidelización aplicando sistemas CRM en la administración de las relaciones con los clientes de las pymes del Cantón Santa Elena - año 2018. [Tesis de Maestría de la Universidad Tecnológica Empresarial de Guayaquil, Ecuador]. Repositorio institucional http://biblioteca.uteg.edu.ec:8080/

Sulbarán, P. (2020). Economía y coronavirus; 7 emprendimientos de Latinoamérica que se reinventaron en medio de la pandemia y están prosperando. [News BBC]. Disponible en: https://www.bbc.com/mundo/noticias-america-latina-52495847

Vara-Horna, A. (2012). Desde la idea hasta la sustentación, siete pasos para una tesis exitosa, un método efectivo para las ciencias empresariales. Instituto de Investigación de la Facultad de Ciencias Administrativas y Recursos Humanos. Universidad de San Martín de Porres. Lima Disponible en: https://idoc.pub/documents/7-pasos-para-una-tesis-exitosa-3no7pmmjmxld

Velasteguí, E. y Toaquiza, I. (2017). Las herramientas tecnológicas al servicio de gestión empresarial y administrativa. Visionario Digital, 1(4), 45-61. DOI: https://doi.org/10.33262/visionariodigital.v1i4.258

Zapata, J., Arango, M. y Adarme, W. (2010). Herramientas tecnológicas al servicio de la gestión empresarial. Revista avances en sistemas e informática, 7(3), 87-101. Disponible en: https://www.redalyc.org/articulo.oa?id=133117498011 


\section{NOTAS BIOGRÁFICAS}

La M.E: Alma Elizabeth Miranda Quiñones, es catedrática del Instituto Tecnológico de Tlalnepantla en el departamento de ciencias económico administrativas, tiene estudios de Licenciatura en Administración Industrial por la U.P.I.I.C.S.A. del Instituto Politécnico Nacional, una maestría en Educación por parte de la U.N.I.D. y actualmente es doctorante en Administración por parte de IEXPRO, ha realizado diversas publicaciones, correo electrónico: almaelizabeth2@gmail.com mirandaae25@gmail.com

El. MAN Ociel Núñez Sánchez, es catedrático Tecnológico de Tlalnepantla en el departamento de ciencias económico administrativas tiene estudios de Licenciatura en Administración Industrial por la U.P.I.I.C.S.A. del Instituto Politécnico Nacional y una maestría en Administración de Negocios por la Universidad Latinoamericana, correo electrónico: ocielns@ tlalnepantla.tecnm.mx

La. M.E. María Magdalena Trejo Lorenzana, es catedrática del Instituto Tecnológico de Tlalnepantla en el departamento de ciencias económico administrativas tiene estudios de Licenciatura en Contaduría, con una maestría en educación por parte de la U.N.I.D. y doctorante en Administración por parte de IEXPRO, ha realizado diversas publicaciones, correo electrónico: maría.tl @ tlalnepantla.tecnm.mx

Dirección institucional de los tres autores: Av. Instituto Tecnológico s/n, Col. La comunidad, Tlalnepantla de Baz, Estado de México, C.P. 54070 